\title{
Coordinated voltage control of a decoupled three-phase on load tap changer transformer and photovoltaic inverters for managing unbalanced networks
}

Hu, Junjie; Marinelli, Mattia; Coppo, Massimiliano ; Zecchino, Antonio; Bindner, Henrik W.

\section{Published in:}

Electric Power Systems Research

Link to article, DOI:

10.1016/j.epsr.2015.10.025

Publication date:

2016

Document Version

Peer reviewed version

Link back to DTU Orbit

Citation (APA):

Hu, J., Marinelli, M., Coppo, M., Zecchino, A., \& Bindner, H. W. (2016). Coordinated voltage control of a decoupled three-phase on load tap changer transformer and photovoltaic inverters for managing unbalanced networks. Electric Power Systems Research, 131, 264-274. https://doi.org/10.1016/j.epsr.2015.10.025

\section{General rights}

Copyright and moral rights for the publications made accessible in the public portal are retained by the authors and/or other copyright owners and it is a condition of accessing publications that users recognise and abide by the legal requirements associated with these rights.

- Users may download and print one copy of any publication from the public portal for the purpose of private study or research.

- You may not further distribute the material or use it for any profit-making activity or commercial gain

- You may freely distribute the URL identifying the publication in the public portal 


\title{
Coordinated voltage control of a decoupled three-phase on-load tap changer transformer and photovoltaic inverters for managing unbalanced networks
}

\author{
Junjie $\mathrm{Hu}^{\mathrm{a}}$, Mattia Marinelli ${ }^{\mathrm{a}, *}$, Massimiliano Coppo ${ }^{\mathrm{b}}$, Antonio Zecchino ${ }^{\mathrm{a}}$, \\ Henrik W. Bindner ${ }^{\mathrm{a}}$ \\ a Center for Electric Power and Energy, Technical University of Denmark, DTU Risø Campus, Frederiksborgvej 399, 4000 Roskilde, Denmark \\ ${ }^{\mathrm{b}}$ Department of Industrial Engineering, University of Padova, Via Gradenigo 6/A, 35131 Padova, Italy
}

\section{A R T I C L E I N F O}

\section{Article history:}

Received 13 May 2015

Received in revised form

24 September 2015

Accepted 21 October 2015

\section{Keywords:}

On-load tap changer

Photovoltaic system

Power transformer

Voltage control

\begin{abstract}
A B S T R A C T
The increasing penetration of fluctuating photovoltaic (PV) generation brings operational challenges for distribution system operators, such as introducing the voltage rise problem. The situation is made worse in the presence of single-phase generation being unevenly connected to the different phases. To address this problem, distribution transformers with single-phase tapping capability, together with reactive power provision of PV systems, are under investigation. This paper presents modeling and analysis of the benefits of coordinated voltage control of a decoupled three-phase on-load tap changer (OLTC) and photovoltaic inverters in a distribution system, for accommodating a greater number of photovoltaic generators in the grid. A $24 \mathrm{~h}$ root-mean-square simulation study is performed in the DigSilent PowerFactory with a $1 \mathrm{~s}$ time step using $10 \mathrm{~min}$ resolution consumption and production profiles on a real Danish distribution grid, as well as the developed dynamic photovoltaic generation and load models. The simulations show that the joint action of the power distribution transformer with OLTC control on each phase, and the reactive power provision of photovoltaic inverters, significantly improves the PV hosting capacity in the analyzed unbalanced scenarios without side effects, such as additional power losses, or significant neutral voltage rises.
\end{abstract}

(C) 2015 Elsevier B.V. All rights reserved.

\section{Introduction}

Network operators nowadays need to face the challenge of ensuring a stable supply voltage in the low-voltage grid, at the same time integrating an increasing number of nonprogrammable distributed energy resources, such as photovoltaic generation, which might raise the risk of violating the permitted voltage band [1-4]. Network operators are being forced into expensive expansion investments, even though the capacities of their grid are far from used up.

Alternatively, as listed in [5] and [6], several solutions have been suggested to deal with the overvoltage phenomena in the presence

Abbreviations: DG, distributed generation; DSO, distribution system operator; MV/LV, medium voltage/low voltage; OLTC, on-load tap changer; PV, photovoltaic; VUF, voltage unbalance factor; One-phase OLTC, decoupled single-phase OLTC that has the possibility of tapping each phase independently.; Three-phases OLTC, the controller changes the taps on 3 phases simultaneously; $Q_{\text {reg, }}$, reactive power provision of photovoltaic inverter.

* Corresponding author. Tel.: +45 20124369.

E-mail address: matm@elektro.dtu.dk (M. Marinelli). of a high level of PV generator penetration in low-voltage grids These methods include: 1) voltage control using reactive power generation from PV inverters [7,8]; 2) voltage control at the LV side of the MV/LV transformer by on-load tap changers (OLTC) [9]; 3) active power derating of PV production in the case of overvoltage conditions [10]; and 4) battery storage/energy buffer at PV generators and MV distribution levels [11]. Each solution is currently investigated by different stakeholders and their feasibility is described in report [6]. In practice, several national standard bodies, such as CEI in Italy [12] and VDE in Germany [13] have updated the connection rules to enable reactive power provision by the inverters interfacing static generators such as PV units, even at low-voltage levels. Different reactive power control methods have been set, most of them relying on local measures on the busbar voltage or the produced active power; they can be summarized as: fixed $\cos \varphi, \cos \varphi(\mathrm{P})$ characteristics, fixed $\mathrm{Q}, \mathrm{Q}(U)$ droop function, and remote set values method.

Coordinated volt/var control has been widely proposed and studied in the literature. In [9], the DG DemoNet project is introduced, the project objective being to develop and test an intelligent voltage control method in an active distribution grid. The 
voltage control method involves the coordination of OLTC operation and reactive power exchange between the distribution system operator and the PV inverter. Several articles [14-17] have been published regarding this project. In [18], a coordinated strategy for voltage control in distribution networks is investigated, where the automatic voltage controller installed at the primary substation cooperates with the DG controllers in setting the active and reactive power set points for generators. In [19], the authors discuss the voltage control with on-load tap changers in medium voltage feeders in the presence of distributed generation. Concerning the OLTC, two kinds of controls are discussed for the conventional distribution grid: the first is based on the local voltage measurement, while the second is intended for remote voltage regulation through line drop compensation (LDC). With the provided background, the authors in [19] studied the effect of both the controls on a distribution network in the presence of DG. Their analysis showed that OLTC and OLTC with LDC are robust against DG, whereas DG can negatively affect the voltage regulation provided by LDC, therefore a proper coordination between DG and LDC is needed to ensure voltage regulation, while enhancing the integration of DG. In [20], the authors presented a proposal for active management of the distribution system that makes use of an innovative controller that coordinates the on-load tap changer action with the regulation of reactive exchanges between DG plants and feeders in MV networks. A similar approach is also presented in [21], where the application of the OLTC control is considered for MV/LV transformers, along with the reactive power control for DG units.

All the aforementioned research activities described, deal with the OLTC's applications on voltage control in three-phase balanced systems, using a synchronized tap changer on the three phases. Other studies $[9,14-17,19,20]$ do not take into account the voltage unbalance effect in low-voltage networks due to the users' single-phase connections, as it usually happens to the PV inverters typically for domestic installations. This kind of connection of the PV could exacerbate the power flow unbalance already existing in these systems due to the unbalanced load connections.

The novelty of this work consists of addressing the voltage control problem through an OLTC capable of operating on the single phases independently; the objective is to evaluate the hosting capacity of a distribution network characterized by high PV penetrations. This study is a further extension of the work presented in [22] in which only the passive network with no PV connections is considered. Highlights of this study include: 1) a coordinated voltage control of a decoupled three-phase on-load tap changer transformer and photovoltaic inverters is proposed for the first time to address the voltage problems of unbalanced distribution networks that are characterized by high PV generations; and 2) details regarding the modeling and control algorithms of OLTC are presented.

The rest of the paper is organized as follows: in Section 2, a general working principle of OLTC in the presence of distributed generation is discussed. Section 3 presents the modeling assumptions and methods for the elements of the distribution system. In Section 4, a Danish low-voltage network adopted as a case study is presented. Simulations are shown in Section 5 to illustrate the performance of the proposed method. In addition, future work on experimental validation of the one-phase OLTC transformer is also described. Finally, conclusions are given in Section 6.

\section{On-load tap changer working principle in the presence of distributed generation}

According to European standard EN 50160, the range of variation of the root-mean-square magnitude of the supply voltage, whether line to neutral, $U_{n}$, or line to line, $U_{c}$, is $U_{n} \pm 10 \%$ or $U_{c} \pm 10 \%$ for at least $95 \%$ of the week. As illustrated in Fig. 1, in practice, a maximum voltage rise of $3-5 \%$ is available to renewable energies in the lowvoltage grid, since the rest is reserved for the medium-voltage grid, considering voltage drops and setting imprecisions.

By using an OLTC transformer instead, the network operator can increase the grid capabilities by dynamically adapting the voltage that decouples the voltages of low- and medium-voltage grids. Referring to the example made in Fig. 1, this may result in an $11 \%$ rather than a $3 \%$ voltage rise being available in the low-voltage grid for feed-in from renewable energies, which is shown in Fig. 2. This kind of action helps to improve the hosting capacity without expensive grid expansion investments.

The transformer is provided with a certain number of taps along one of the windings in order to adjust the turns ratio between the primary and secondary sides. A tap changing device that makes the physical change in the tap position during on-load operation is

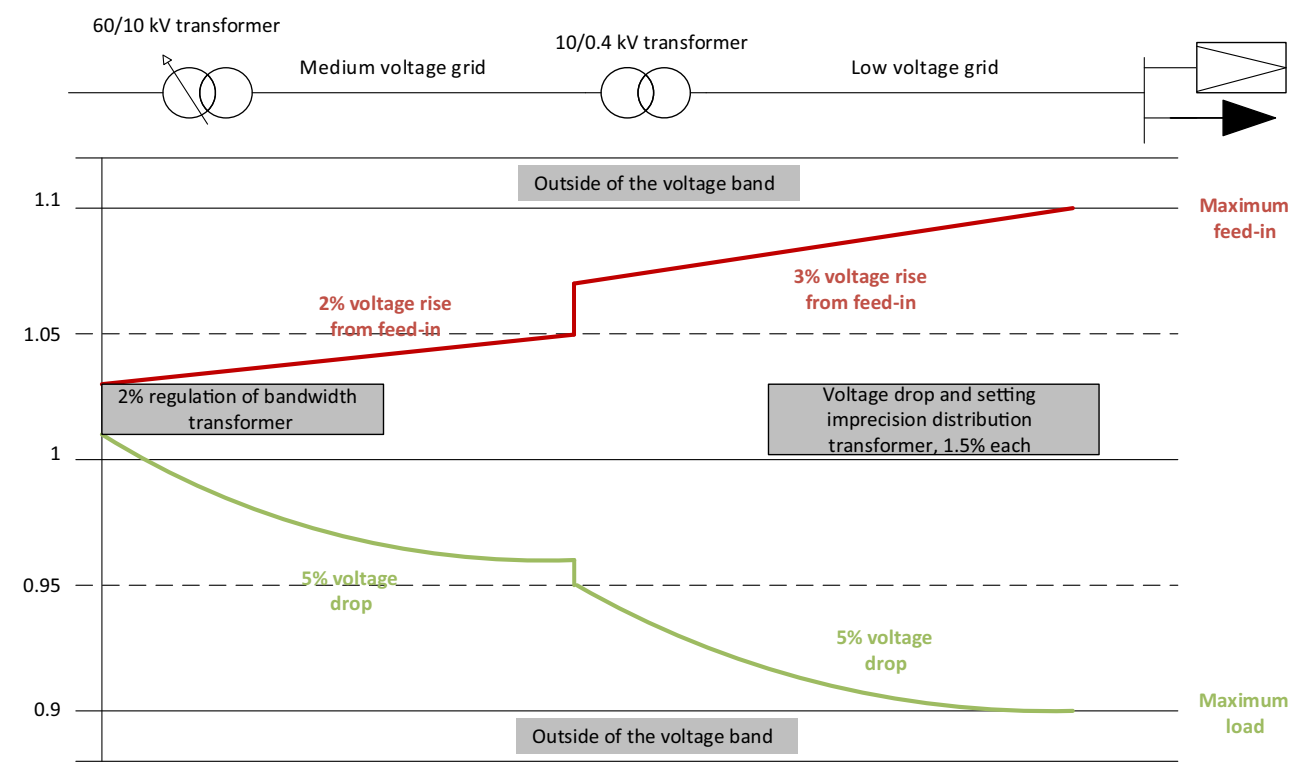

Fig. 1. Potential problems faced by the network operator without OLTC. 


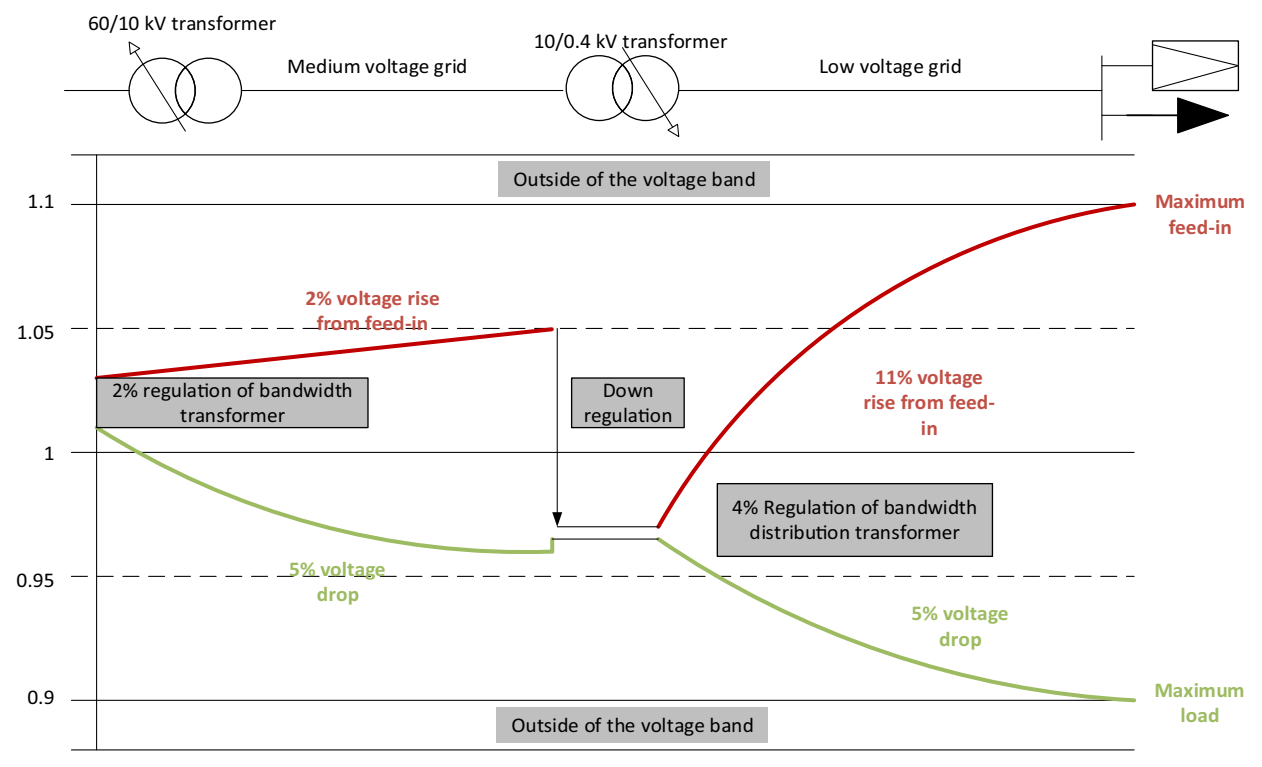

Fig. 2. Advantages offered to the network operator in the presence of OLTC.

placed on the winding with a higher voltage to minimize the current to be switched, and can be "off-circuit" or "on-load" type. OLTC are usually equipped with oil-immersed transformers connecting HV networks to MV systems. Since the majority of power distribution companies stipulated a voltage variation of $\pm 10 \%$ in the power contract, the tap changer is provided with an equivalent range of voltage regulation of $\pm 10 \%$ in 16 or 32 steps: a 16 steps tap changer provides $1.25 \%$ voltage change at each step, while a 32 steps tap changer provides $0.625 \%$ voltage change at each step; thus the latter is preferred when a more precise control is needed. In this study, more details regarding the modeling and control algorithm of the OLTC are introduced in Section 3.1.

\section{Modeling the distribution system's elements}

The system elements involved in the analysis have been modeled and simulated with the software DigSilent PowerFactory $[23,24]$, in order to test the effects of the on-load tap changing capability at the MV/LV transformer on an unbalanced low-voltage network. The following sections discuss the implementation of the components involved in the analysis.

\subsection{OLTC modeling}

The main focus of this paper lies on the MV/LV transformer one-phase tap changing capability, aimed at mitigating voltage deviations at a certain busbar (e.g., the point of common coupling
(PCC) at the transformer's secondary busbar). The three-phase Delta-Wye transformer connecting the LV system under test to the MV network has been modeled as three single-phase transformers, each independently controlled, in order to have a one-phase tap changing capability. The control scheme for the OLTC transformers is shown in Fig. 3. We note that the control scheme is also applicable to three-phase OLTC transformers. Two regulating options are investigated in order to compare and verify the effectiveness of the control: three-phase OLTC transformer and one-phase OLTC transformer. The first regulation option consists of operating the three OLTC synchronously on the three phases, referring to the voltage value on one phase (in this study phase a): this operation is expected from a standard OLTC, and the choice of selecting just one phase voltage value for the control would result in a costeffective solution for the device (since it is supposed to work with a balanced voltage triplet and it requires just one-phase voltage measurement). The one-phase OLTC control is then taken into account, allowing the three tap-changing devices to operate independently, referring each to the respective voltage measurement.

Each tap position variation is obtained through a proportional controller behaving as for the characteristics shown in Fig. 4, continuously changing the tap position depending on the voltage measured at the controlled bus. In Fig. 4, two examples of linear characteristics are shown: the red curve shows the tapping range between -1 and 1 , which means the voltage is regulated within $\pm 2.5 \%$ of the nominal voltage; the blue curve illustrates the tapping range between -2 and 2 , which means the voltage is regulated within $\pm 5 \%$ of the nominal voltage.

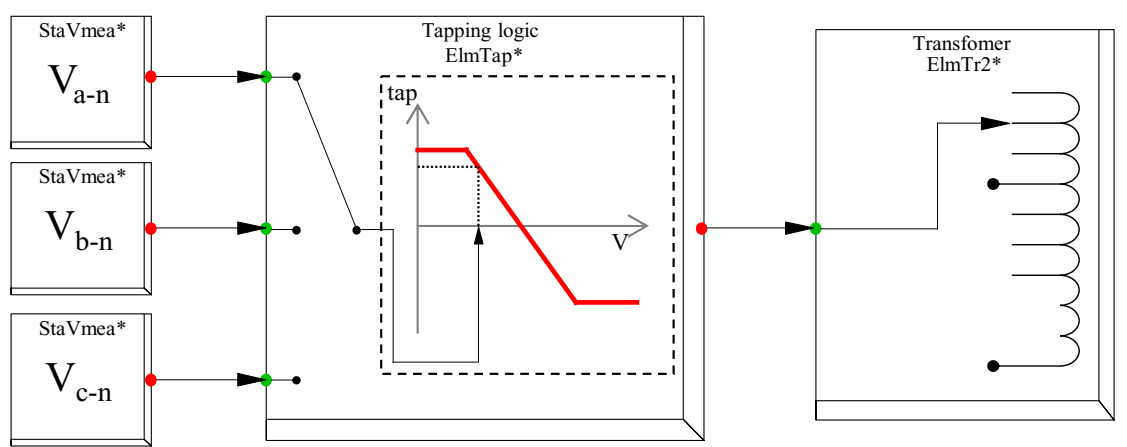

Fig. 3. Control scheme for three-phase and one-phase OLTC transformers. 


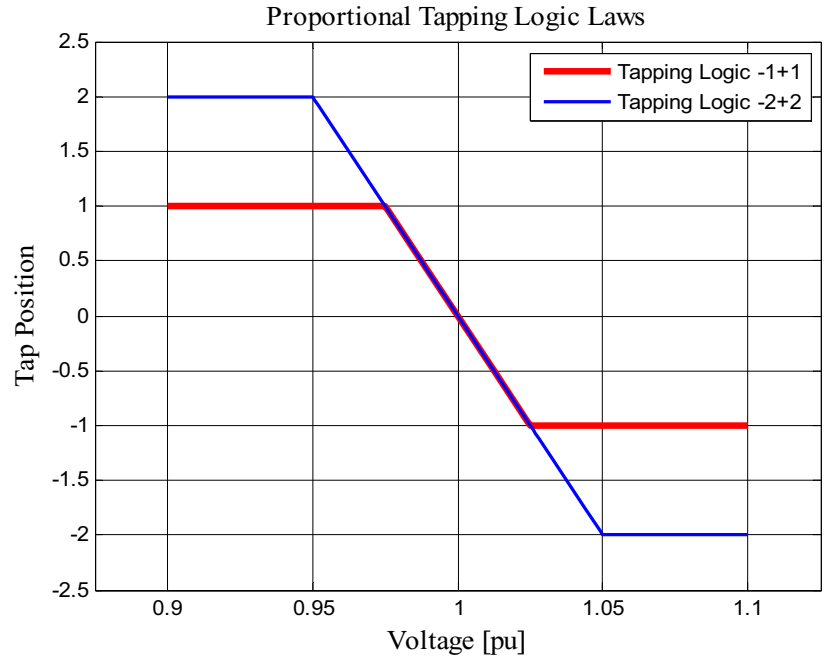

Fig. 4. Characteristics of the transformer proportional controllers.

\subsection{Load modeling}

Loads have been represented as constant impedance units, varying their absorption through the $24 \mathrm{~h}$ time window considered for the simulations according to real measurements coming from a real low-voltage network. Field measurements of voltages, currents, and active power coming from a real Danish LV network have been used to characterize the load profiles. The measurements, taken on the three phases at several busbars of the system, have a $10 \mathrm{~min}$ resolution and cover a $24 \mathrm{~h}$ interval. The time-dependent power profiles obtained processing the field data are then applied to the single-phase loads through the scheme shown in Fig. 5, where the active and reactive power values are read from an external file and applied to the single-phase load.

The load, represented in Fig. 5 by a block (LoadSlot) is then modeled as for Eqs. (1-2), which gives a general definition of the active and reactive powers' voltage dependencies.

$P_{\mathrm{ZIP}}=P_{0}\left[a_{1}\left(\frac{V}{V_{0}}\right)^{2}+a_{2}\left(\frac{V}{V_{0}}\right)+a_{3}\right]$

$Q_{\mathrm{ZIP}}=Q_{0}\left[a_{1}\left(\frac{V}{V_{0}}\right)^{2}+a_{2}\left(\frac{V}{V_{0}}\right)+a_{3}\right]$

Eqs. (1) and (2) refer to an active and reactive powers definition also known as ZIP [25] model, where three terms $a_{1}, a_{2}$, and $a_{3}$ represent the shares of the constant impedance, constant current, and constant power contributions; $P_{0}$ and $Q_{0}$ are the active and reactive powers at rated voltage $V_{0} ; P_{\text {ZIP }}$, and $Q_{\text {ZIP }}$ are the active and reactive powers at operating voltage $V$. In this work, for the dynamic

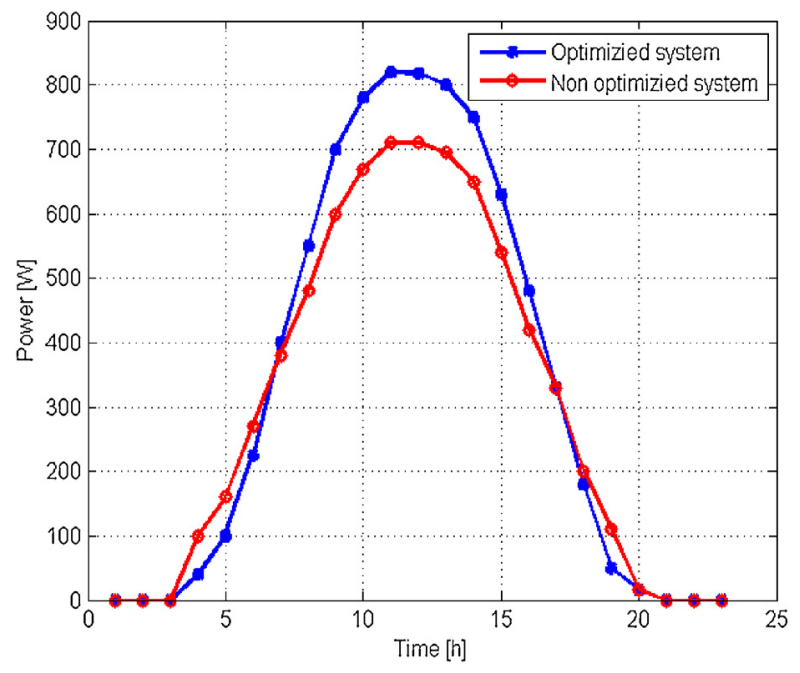

Fig. 6. Output power for the photovoltaic unit under clear-sky conditions.

simulations, the loads have been modeled as constant impedances, so the three components have been set to the following values: $a_{1}=1, a_{2}=0$, and $a_{3}=0$. Fig. 5 shows the process of applying variable power set points, i.e., $P_{0}$ and $Q_{0}$ in Eqs (1) and (2), to the load models during the dynamic simulation.

\subsection{PV generation profile modeling}

One aim of this work is to analyze the effects of the on-load tap changer on the low-voltage network in presence of different degrees of PV penetration. In the process, the PV generation profiles are characterized by the ideal day active power profile shown in Fig. 6.

In Fig. 6, two photovoltaic active power output profiles are shown for a $24 \mathrm{~h}$ interval under clear-sky conditions [6]. The two curves refer to different PV orientations: the blue curve, representing the best case, is the output obtainable from an aggregation of systems pointing south with a $45^{\circ}$ inclination, while the red curve, considering of a more realistic case (also used for this work), is related to a group of $10 \mathrm{PVs}$ with different orientations (East-West) and inclinations $\left(30-45^{\circ}\right)$. Clear-sky conditions are considered in this study and can represent the "worst case" in terms of introducing the voltage rise problem to the distribution network, since it generates the maximum power. Other effects due to reduced PV production can be observed in earlier/later hours of the day in the simulation. By analyzing the control effect of the OLTC under the idea sunny day, it is ensured that the OLTC can handle different PV production levels.

The PV units in this work have been modeled as "active loads" with constant power, assumption justified by the fact that PV

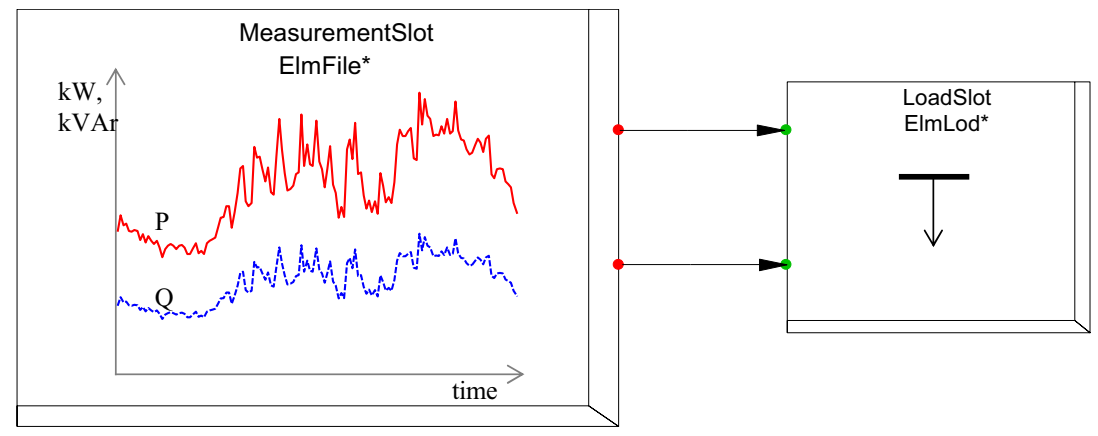

Fig. 5. Load dynamic model. 


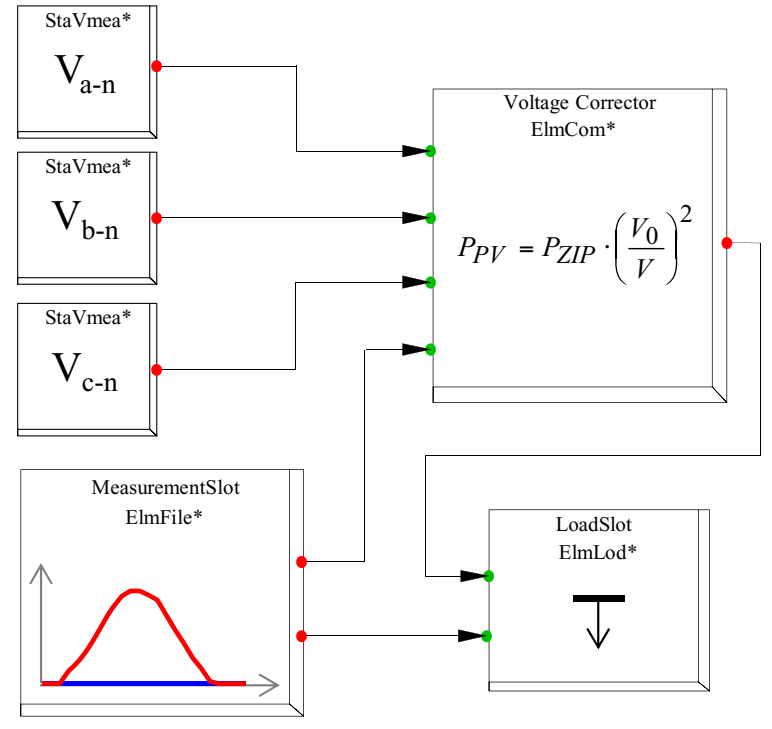

Fig. 7. Photovoltaic unit dynamic model.

generators are interfaced to the distribution grid through inverters controlling the active power output.

Since, as mentioned previously, the simulation tool considers the shunt elements (i.e., loads, in this case) as constant impedances, a correction to the power set point assigned to the active loads is needed. This feature is obtained through the control scheme reported in Fig. 7, which is derived from the one in Error! Reference source not found. with the addition of a "Voltage Correction" block that implements Eq. (3), where $P_{\mathrm{ZIP}}$ and $P_{\mathrm{PV}}$ are respectively the active power calculated as for Eq. (1) with $a_{1}=1, a_{2}=0$, and $a_{3}=0$, and the corrected active power output of the PV unit:

$P_{\mathrm{PV}}=P_{\mathrm{ZIP}} \cdot\left(\frac{V_{0}}{V}\right)^{2}=P_{0}$

\section{4. $P V$ reactive power control modeling}

Since the Danish grid code does not provide for any technical guidelines about the reactive power management by the distributed generation plants connected to the LV network, it is reasonable to expect that future Danish requirements will
Table 1

Total load energy (24h) and mean power values.

\begin{tabular}{llll}
\hline & Phase a & Phase b & Phase c \\
\hline Energy [kWh] & 295.5 & 201.2 & 242.4 \\
Mean Power [kW] & 12.4 & 8.4 & 10.2 \\
\hline
\end{tabular}

experience harmonization with other European regulations, such as the Italian and German ones-respectively CEI 0-21 [12] and VDE-AR-N 4105 [13]. For this reason, in this work it has been decided to start from those technical standards in order to implement a local control characteristic based on local measurements of the voltage and the active power produced. The characteristic adopted in this work, which is derived from the one presented in [4], is shown in Fig. 8 and includes both voltage and active power dependence, as described in Eq. (4):

$Q=f(V, P)$

As can be seen from Fig. 8, the reactive power contribution by inverters is set in order to reduce the voltage deviation at the connection busbar, so when a voltage rise is detected, the inverter will start operating as an inductor (i.e., absorbing reactive power), and conversely when a voltage sag occurs, the inverter will start injecting reactive power, operating as a capacitor. The characteristic has a dead-band between $0.99 \mathrm{Un}$ and $1.01 \mathrm{Un}$, while the voltage limits have been set to $\pm 10 \%$ of the nominal voltage Un. The reactive power limit has been set to 0.5 p.u. (with respect to the inverter's rated apparent power) [12,13].

\section{A Danish low-voltage network}

The models described in Section 3 have been implemented in a real low-voltage grid, provided by DONG Energy Els distribution company [26]. The network consists of 33 single-phase loads and 18 single-phase PVs that are connected to the MV network through a $10 / 0.4 \mathrm{kV}$ transformer as shown in Fig. 9. The MV grid connection has been represented as a voltage source with a 20 MVA short circuit power.

Field measurements of voltages, currents, and powers of the three phases of the busbars have been considered in order to obtain the consumption profiles for each of the 33 single-phase loads during a $24 \mathrm{~h}$ interval, resulting in about $740 \mathrm{kWh}$, with a mean power of $30.8 \mathrm{~kW}$. The total energy and mean power for each phase are reported in Table 1.

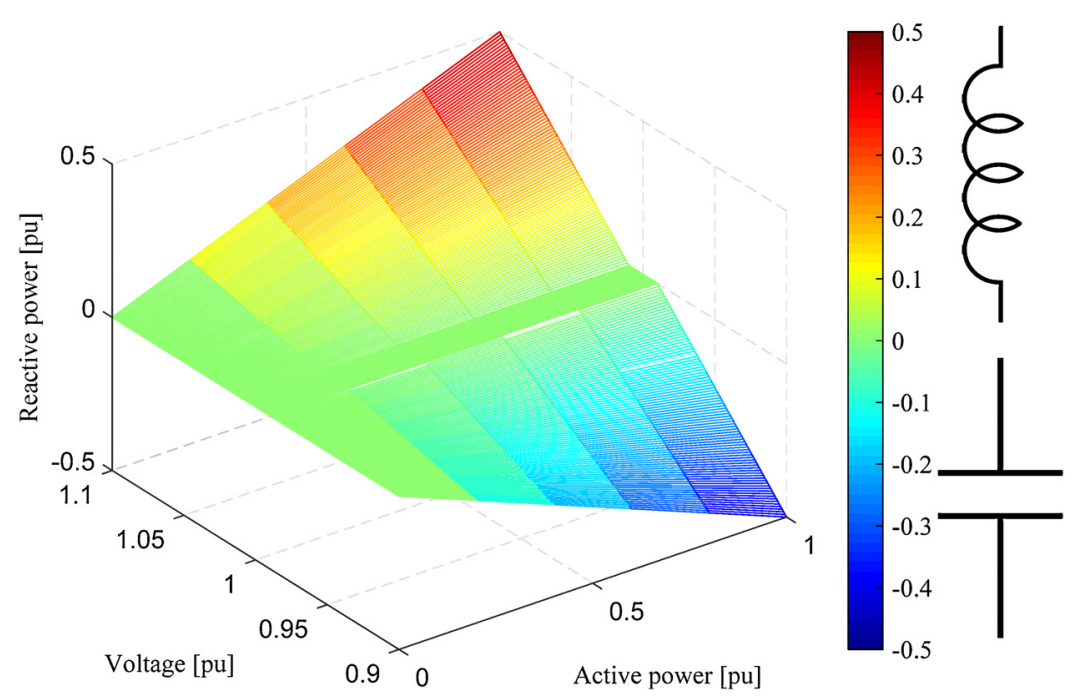

Fig. 8. Reactive power control characteristic for PV inverters. 
Table 2

PV connection assumptions in the studied network.

\begin{tabular}{|c|c|c|c|}
\hline $\begin{array}{l}\text { PV penetration } \\
\text { level [\%] }\end{array}$ & $\begin{array}{l}\text { Total PV power for a LV network with } 70 \\
\text { customers [kVA], one PV inverter } 5 \mathrm{kVA}\end{array}$ & $\begin{array}{l}\text { Phase connections (A, B, C with } \\
\text { different penetration \%) }\end{array}$ & Number of customers \\
\hline 40 & 140 & A, B, C $(50,30,20)$ & 28 \\
\hline 50 & 175 & A, B, C $(50,30,20)$ & 35 \\
\hline 60 & 210 & A, B, C $(50,30,20)$ & 42 \\
\hline 70 & 245 & A, B, C $(50,30,20)$ & 49 \\
\hline
\end{tabular}

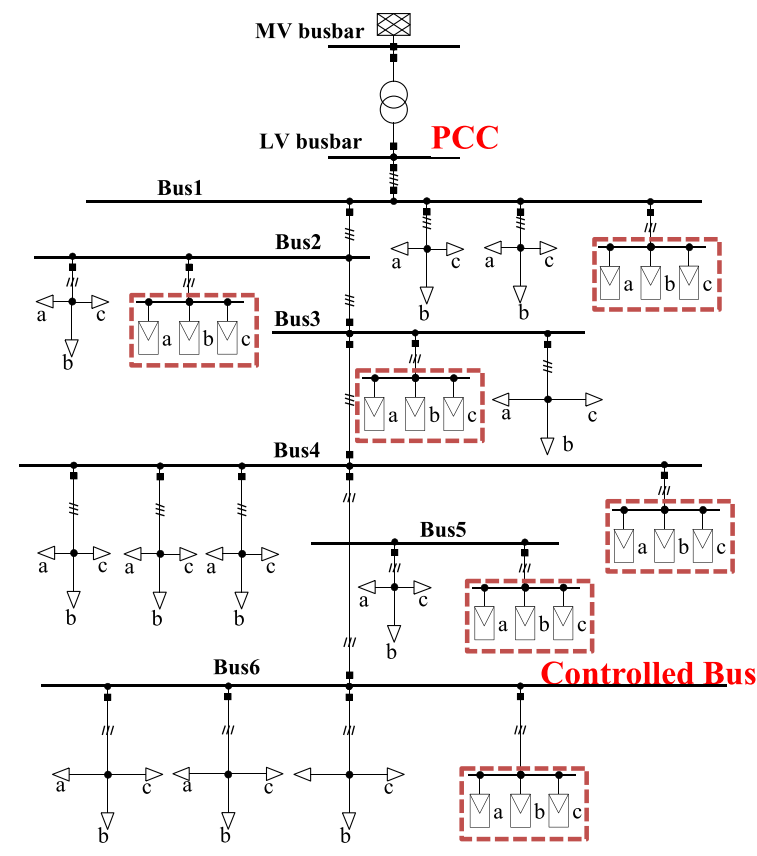

Fig. 9. Danish LV network layout with PV connections.

In order to evaluate the effects of the one-phase OLTC control in the presence of different degrees of DG integration, the PV penetration level is introduced in this study and is defined by the ratio of the number of households that have PVs and the total number of households [6]. Assuming that the grid has installed PV inverter capacity of $350 \mathrm{kVA}$ (i.e., $100 \% \mathrm{PV}$ penetration) and a PV unit size of $5 \mathrm{kVA}$, which is expected to be an affordable size for householders under the present legislative framework, the total installed PV power in each simulation scenario can be derived using Eq. (5):

$S_{\mathrm{TOT}}=\left(n_{\mathrm{CUST}} \cdot k_{\mathrm{PV} \%}\right) \cdot S_{r}$

where

$S_{\mathrm{TOT}} \quad$ total apparent power installed in the network;

$n_{\text {CUST }}$ total number of customers connected on this low-voltage network, set to 70 .

$k_{\mathrm{PV} \%} \quad$ percentage of PV penetration in the network;

$S_{r} \quad$ apparent power of the single PV unit, chosen as $5 \mathrm{kVA}$.

\section{Simulations}

\subsection{Definition of simulation cases and scenarios}

The investigation of the effects of the OLTC operation presented in Section 3 has been made over a $24 \mathrm{~h}$ time period, considering different control options in the following five cases:

- Base case: the transformer is not equipped with any tap changing device;
Table 3

PV power distribution over the six buses of each phase for $70 \%$ scenario.

\begin{tabular}{llll}
\hline & Phase a & Phase b & Phase c \\
\hline Bus 1 & $20 \mathrm{kVA}$ & $15 \mathrm{kVA}$ & $10 \mathrm{kVA}$ \\
Bus 2 & $20 \mathrm{kVA}$ & $15 \mathrm{kVA}$ & $10 \mathrm{kVA}$ \\
Bus 3 & $15 \mathrm{kVA}$ & $10 \mathrm{kVA}$ \\
Bus 4 & $20 \mathrm{kVA}$ & $10 \mathrm{kVA}$ & $10 \mathrm{kVA}$ \\
Bus 5 & $20 \mathrm{kVA}$ & $10 \mathrm{kVA}$ & $5 \mathrm{kVA}$ \\
Bus 6 & $20 \mathrm{kVA}$ & $10 \mathrm{kVA}$ & $5 \mathrm{kVA}$ \\
\hline
\end{tabular}

- three-phase OLTC: the OLTC is controlled synchronously on the three phases, taking as a reference the phase-neutral voltage at phase a.

- one-phase OLTC: the control is set to independently commute the tap positions on each phase winding.

- $\mathrm{Q}_{\text {reg }}$ : reactive power provision from PV inverters is studied to regulate the voltage. The purpose is to compare the local control performance of the inverters to the OLTC control solution.

- one-phase OLTC with $\mathrm{Q}_{\text {reg }}$ : besides one-phase OLTC, reactive power provision from PV inverters is added to address the voltage deviation problem and to evaluate the joint control effect.

With the aim of reducing the voltage deviation from the nominal value at the outermost busbar of the feeder, the voltage measurements used as an input for the OLTC control are taken from Bus 6 as highlighted in Fig. 9.

The control options of the five cases have been applied to four PV penetration scenarios as shown in Table 2, obtained by progressively increasing the installed power in order to check the grid's hosting capacity. The PV penetration percentages selected for the simulation scenarios start from $40 \%$ up to $70 \%$ with respect to the maximum installed power of $350 \mathrm{kVA}$ (i.e., 70 households with $5 \mathrm{kVA}$ power). The $40 \% \mathrm{PV}$ penetration was chosen as the starting scenario, since in that case the distribution network approaches the voltage limitations, while $70 \%$ of PV penetration is the maximum grid hosting capacity reachable by adopting the control options.Note that for each PV penetration level, PV power is first distributed on each phase based on the numbers defined in Table 2. Then, the PV power of each phase is evenly distributed to all six buses shown in Fig. 9. For example, the total installed PV power is $245 \mathrm{kVA}$ for the scenario $70 \%$ and it is distributed as shown in Table 3 .

\subsection{Simulation results}

In the following section, an analysis of the simulation results is given by verifying several indices relating to the hosting capacity of the LV grid, in particular: the voltage deviation from nominal value, the voltage unbalance factor (VUF), and the neutral potential and total energy loss during the simulated $24 \mathrm{~h}$.

The degree of voltage unbalance factor is quantified as:

$\mathrm{VUF} \%=\frac{V_{-}}{V_{+}} \times 100$

According to [27], Eq. (6) permits the evaluation of the Voltage Unbalance Factor (VUF), defined as the ratio between the negative and positive sequence voltage components in percent. 
Table 4

Summary of the simulation results.

\begin{tabular}{|c|c|c|c|c|c|}
\hline Scenario & Case & Max phase voltages deviation at bus 6 & Max VUF at bus 6 & Max neutral potential (\%Vn) & Total losses [kWh] \\
\hline \multirow[t]{5}{*}{ PV 40\% } & Base case & $-4.0 \% /+7.0 \%$ & $1.00 \%$ & $2.50 \%$ & 11.61 \\
\hline & Three-Phase OLTC & $-6.5 \% \mid+4 \%$ & $1.10 \%$ & $2.50 \%$ & 12.22 \\
\hline & One-Phase OLTC & $-2.0 \% /+3.5 \%$ & $1.30 \%$ & $2.50 \%$ & 12.15 \\
\hline & Qreg & $-4.0 \% /+5.2 \%$ & $1.24 \%$ & $2.15 \%$ & 11.73 \\
\hline & One-Phase OLTC $+Q_{\text {reg }}$ & $-2.0 \% \mid+3.1 \%$ & $1.42 \%$ & $2.20 \%$ & 12.12 \\
\hline \multirow[t]{5}{*}{ PV 50\% } & Base case & $-5.5 \% \mid+11.0 \%$ & $1.40 \%$ & $3.75 \%$ & 21.45 \\
\hline & Three-Phase OLTC & $-11.0 \% /+6.5 \%$ & $1.40 \%$ & $3.80 \%$ & 23.59 \\
\hline & One-Phase OLTC & $-2.5 \% \mid+5.5 \%$ & $1.80 \%$ & $3.85 \%$ & 23.31 \\
\hline & Qreg & $-3.9 \% \mid+7.3 \%$ & $2.00 \%$ & $2.75 \%$ & 20.90 \\
\hline & One-Phase OLTC $+Q_{\text {reg }}$ & $-2.2 \% \mid+4.7 \%$ & $2.32 \%$ & $3.30 \%$ & 21.02 \\
\hline \multirow[t]{5}{*}{ PV 60\% } & Base case & $-6.0 \% \mid+11.0 \%$ & $1.50 \%$ & $3.95 \%$ & 28.41 \\
\hline & Three-Phase OLTC & $-11.0 \% /+7.0 \%$ & $1.50 \%$ & $4.00 \%$ & 31.62 \\
\hline & One-Phase OLTC & $-3.0 \% \mid+7.0 \%$ & $1.80 \%$ & $4.05 \%$ & 31.03 \\
\hline & $\mathrm{Q}_{\text {reg }}$ & $-4.0 \% \mid+7.8 \%$ & $2.22 \%$ & $3.00 \%$ & 22.99 \\
\hline & One-Phase OLTC $+Q_{\text {reg }}$ & $-2.3 \% \mid+5.2 \%$ & $2.63 \%$ & $3.58 \%$ & 29.22 \\
\hline \multirow[t]{5}{*}{ PV 70\% } & Base case & $-4.0 \% \mid+13.0 \%$ & $1.63 \%$ & $4.08 \%$ & 38.10 \\
\hline & Three-Phase OLTC & $-10.1 \% /+8.8 \%$ & $1.65 \%$ & $4.30 \%$ & 43.07 \\
\hline & One-Phase OLTC & $-2.0 \% \mid+8.8 \%$ & $2.01 \%$ & $4.42 \%$ & 41.73 \\
\hline & $\mathrm{Q}_{\mathrm{reg}}$ & $-4 \% \mid+8.1 \%$ & $2.56 \%$ & $3.55 \%$ & 41.22 \\
\hline & One-Phase OLTC $+Q_{\text {reg }}$ & $-2.0 \% /+6.0 \%$ & $3.42 \%$ & $4.15 \%$ & 42.30 \\
\hline
\end{tabular}

The results are summarized in Table 4. For each PV penetration scenario, the values for the mentioned indices under the five cases are presented. Particular emphasis is placed on the voltage deviation and VUF values, considering respectively, $\pm 10 \% \mathrm{~V}_{\mathrm{n}}$ and $2 \%$ as limits for acceptable network operation, and, for this reason, the values are highlighted in red when overcoming those bounds. As shown in Table 4, in the case of $40 \%$ PV penetration, the PVs in the grid are safely integrated without any additional control, since the voltage deviation at Bus 6 is contained between -4 and $+7 \%$ of the nominal voltage and VUF remains below $1 \%$ during the entire period.

The application of the three-phase OLTC control allows the reduction of the voltage deviations within a total $10.5 \%$ $(-6.5 \% /+4 \%)$ band around the nominal voltage $(11.0 \%$ in the Base case). Adopting the one-phase OLTC control, instead, the voltage deviation is reduced within a $5.5 \%$ band around the nominal voltage. However, one-phase OLTC control leads to a small increase in the VUF level ( $+0.3 \%$ respect to the base case). Qreg control improves the voltage profile compared to the base case. The adoption of the one-phase OLTC with $Q_{\text {reg }}$ regulation strategy allows the best outcome in terms of voltage magnitude regulation, even though with the PV $40 \%$ scenario and the adoption of this control method, the VUF increases by $0.42 \%$ with respect to the base case but still does not exceed the permitted value of $2 \%$.

Under the modeling hypothesis discussed in Section 3, both controls do not affect the neutral potential, while they tend to increase the energy losses by around $0.6 \mathrm{kWh}(+5 \%$ compared to the base case losses). This effect can be explained by noticing that the PVs are modeled as constant power units (i.e., current is inversely proportional to the voltage) and that during the PV production period, the injected power is much greater than the load absorption.

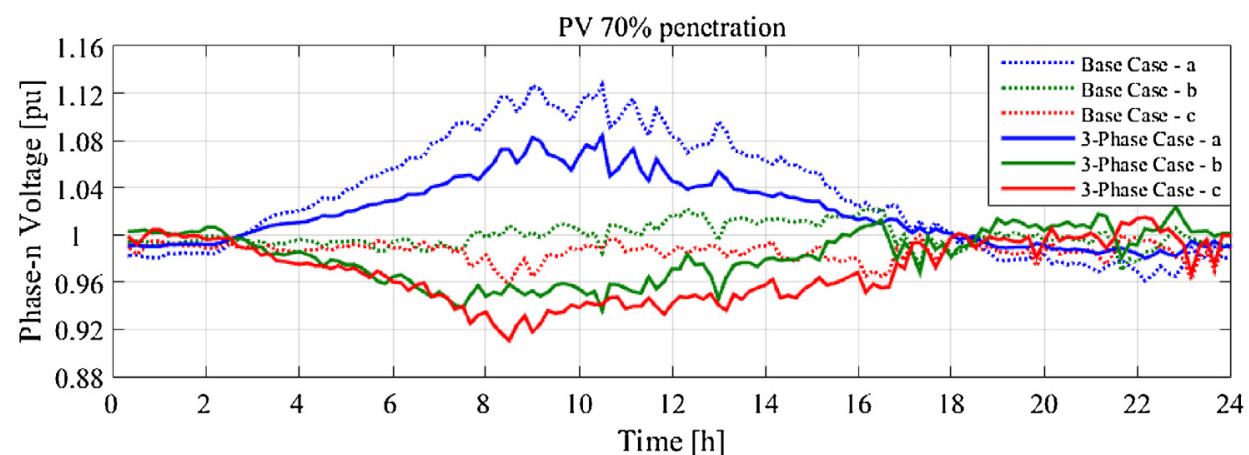

(a)

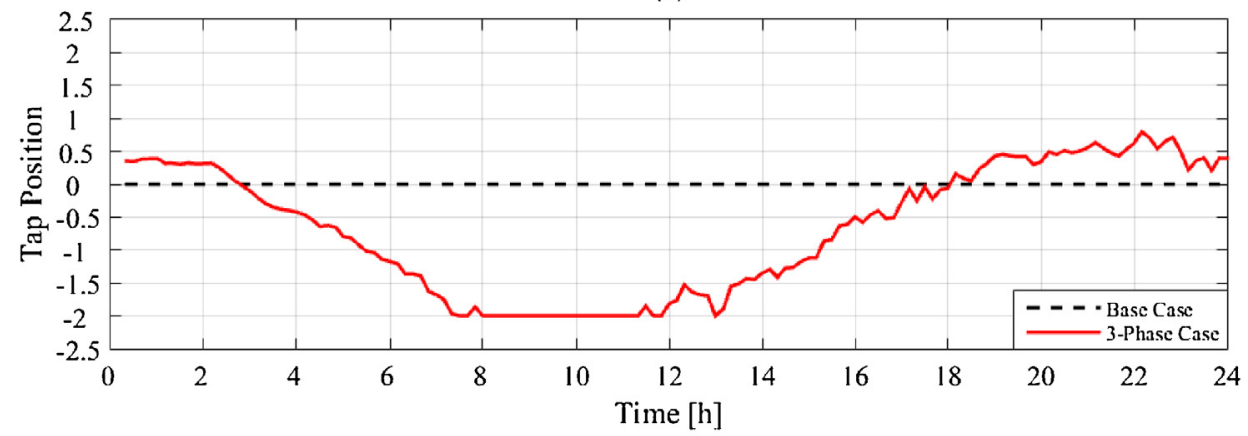

(b)

Fig. 10. Voltage measurements at bus 6 and tap positions in three-phase case and base case scenario. 
On the other hand, when the PVs are not producing, current and voltage are directly proportional, due to the constant impedance loads model. As a result, since the reduction of the voltage deviation is more sensible during the PV production period, the effect of inverse proportionality between voltage and current is, on average, more relevant during the entire period, resulting in an overall increase of the current flowing through the network branches and then leading to greater losses. This effect is even more intense when including the independent tap changing strategy, since the voltage variation is greater in each phase.
Table 4 shows that by increasing the PV penetration percentage to $50 \%, 60 \%$, and $70 \%$, the voltage deviation at the controlled bus becomes unacceptable, exceeding the $\pm 10 \% \mathrm{Vn}$ band. This effect remains when applying the three-phase OLTC strategy, since the control was designed to refer to the voltage of phase a. Fig. 10a shows that increasing the PV penetration to $70 \%$ results in a voltage increase in phase $a$, while phases $b$ and $c$ have their voltages around the nominal value. With the three-phase OLTC control option, the voltage of phase a decreases, while the voltages of phase $b$ and $c$ deviate from their nominal values, since the tendency is to reduce

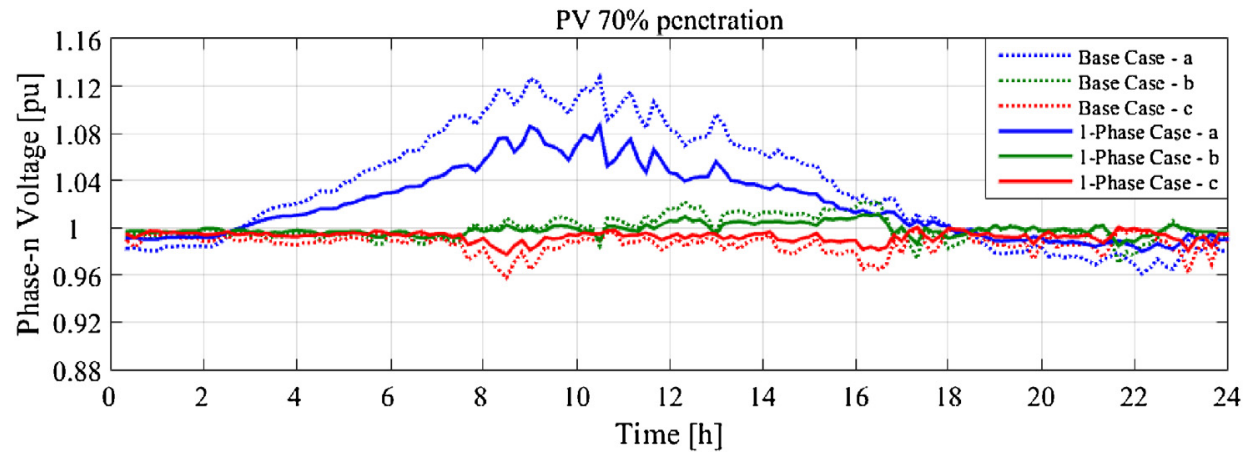

(a)

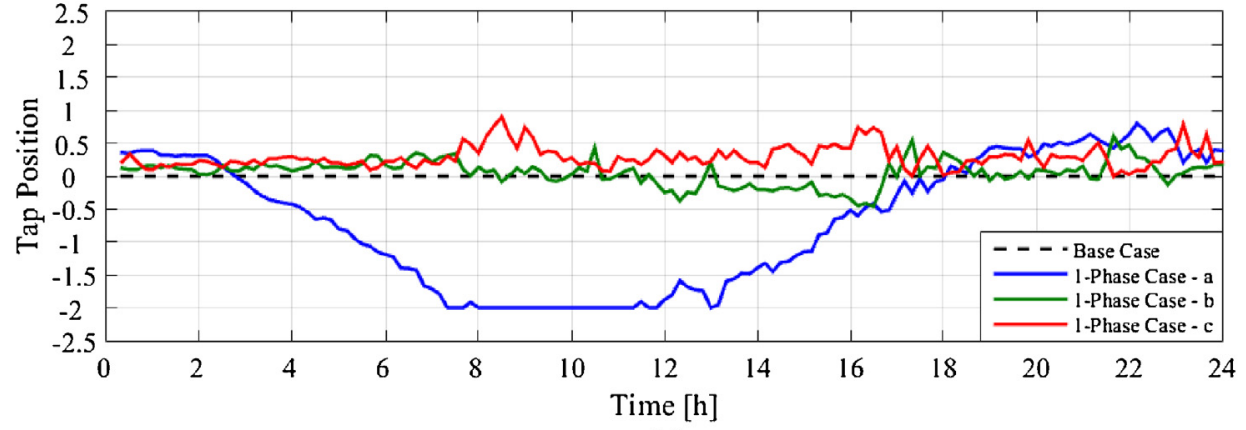

(b)

Fig. 11. Voltage measurements at bus 6 and tap positions in one-phase case and base case scenario.
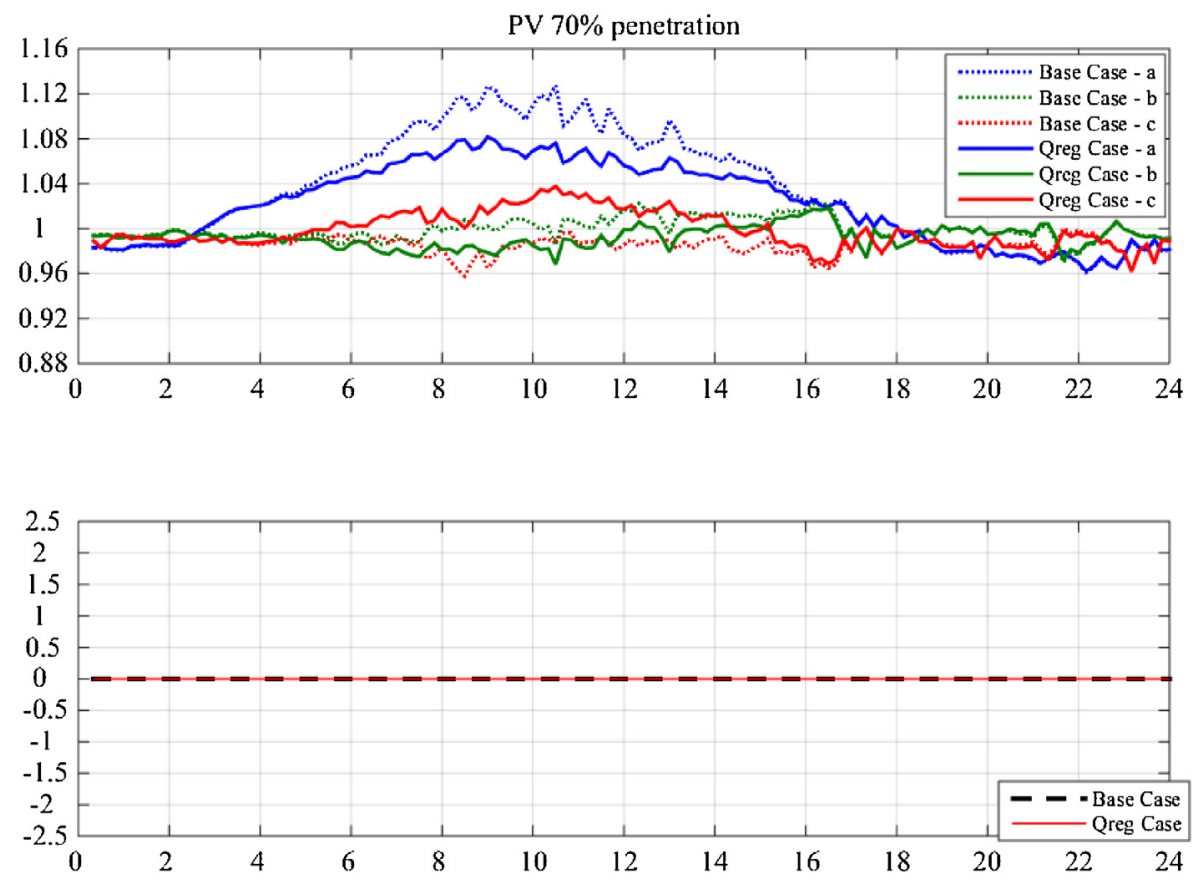

Fig. 12. Voltage measurements at bus 6 and tap positions in $Q_{\text {reg }}$ case and base case scenario. 


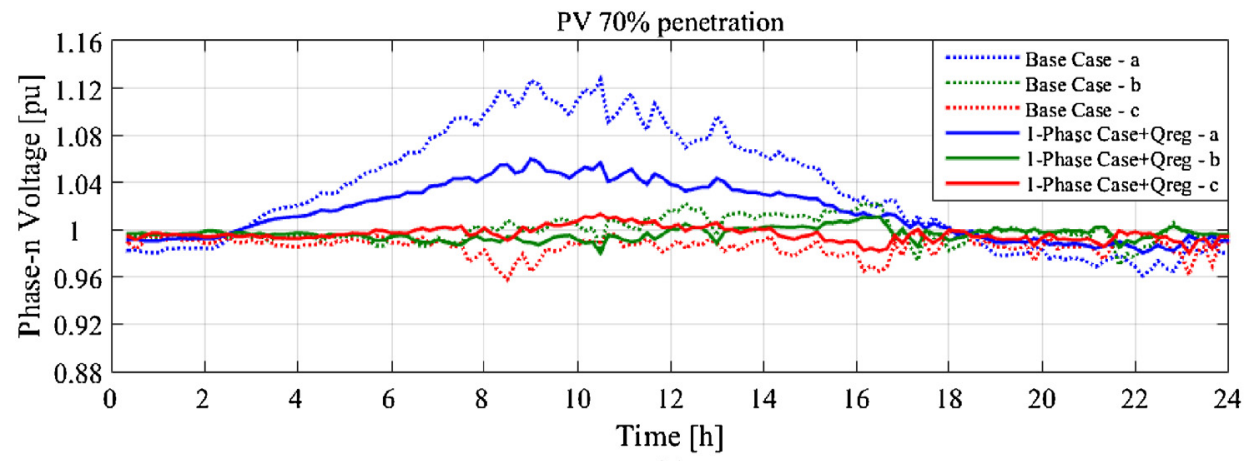

(a)

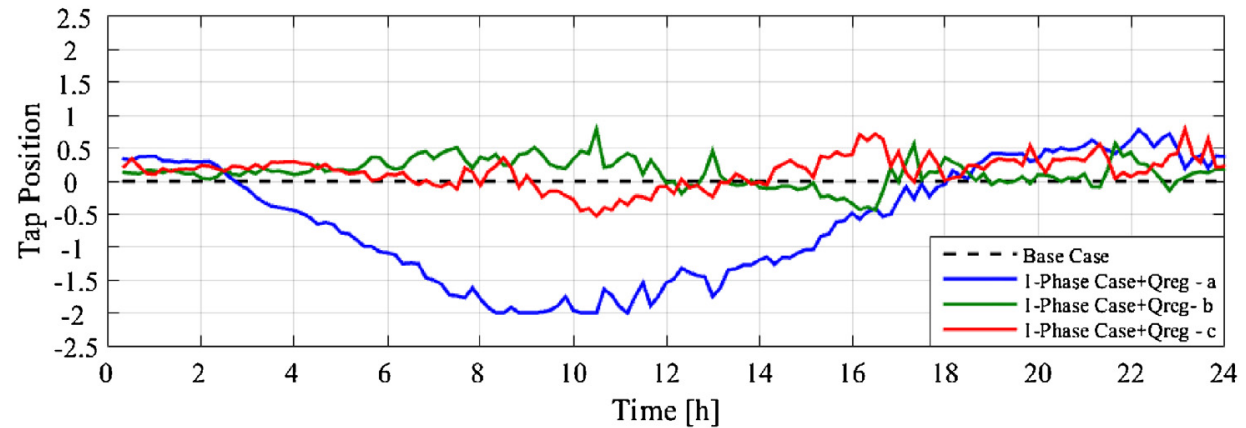

(b)

Fig. 13. Voltage measurements at bus 6 and tap positions in one-phase case with $Q_{\text {reg }}$ and base case scenario.

the overvoltage in phase a as well as to reduce the other two phases' voltages. The corresponding tap positions are shown in Fig. 10b.

The voltage values at Bus 6, along with the tap positions under the 70\% PV penetration scenario are shown in Fig. 11 for the onephase OLTC control. From Fig. 11, it can be seen that the adoption of the one-phase OLTC control option by itself can help mitigate the voltage deviation from $17 \%$ to $11.8 \%$ overall, preventing the voltage to exceed the $\pm 10 \% \mathrm{Vn}$ band. In between, Fig. 12 shows the voltage profile obtained at Bus 6 when adopting the Q Q reg control solution and the results show that voltages are kept in the safe band and the overall voltage devation is $12.1 \%$. In Fig. 13, the voltage and relative tap position in the same $\mathrm{PV}$ penetration scenario are referred to the case including both the one-phase OLTC and reactive power controls, showing that the voltage deviation at bus 6 could be further reduced to about $8 \%$.

Regarding the voltage unbalance, Table 4 shows that the VUF tends to rise in the one-phase OLTC control when increasing the $\mathrm{PV}$ penetration. The effect is even more intense when including the local reactive power control to the DG units. This is because the positive and negative sequence magnitudes are subject to opposite trends (i.e., the positive sequence tends to decrease, while the negative sequence increases). In particular, the local control by DGs causes the uncoordinated injection of different values of reactive power, negatively affecting the VUF. On the other hand, the presence of this local action helps to mitigate the neutral conductor's current, resulting in a smaller neutral potential.

The VUF values obtained in the 70\% PV penetration scenario are shown in Fig. 14 associated with each of the cases presented before: as already mentioned, the control solutions could worsen the maximum VUF value from $2 \%$ to about $3.5 \%$.

\subsection{Future work on experimental validation of the one-phase on-load tap changer transformer}

This study has shown that the decoupled three-phase onload tap changer transformer can effectively regulate the voltage

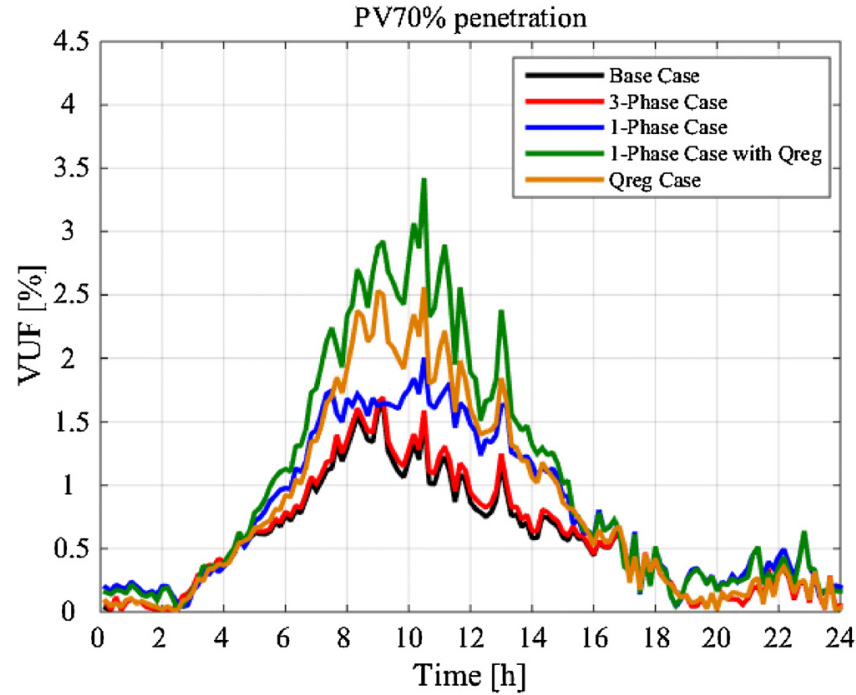

Fig. 14. VUF at bus 6 in five control options under the $70 \% \mathrm{PV}$ penetration scenario.

introduced by the PV installation. To validate the control performance of the one-phase OLTC transformer, the device will be experimentally tested using the SYSLAB-PowerLabDK experimental facility at the DTU Risø Campus (http://www.powerlab.dk/ facilities/syslab.aspx). Fig. 15 shows the internal structure of the one-phase OLTC transformer whose regulation range is $\pm 10 \%$ of input voltage. The rated power of the transformer is $3 \times 11.66 \mathrm{kVA}$. The device will be tested in the configuration illustrated in Fig. 16. The test will be performed with different generations and load conditions by measuring and analyzing the phase-neutral voltage, neutral to ground voltage, currents in the line, and voltage unbalance factor. 


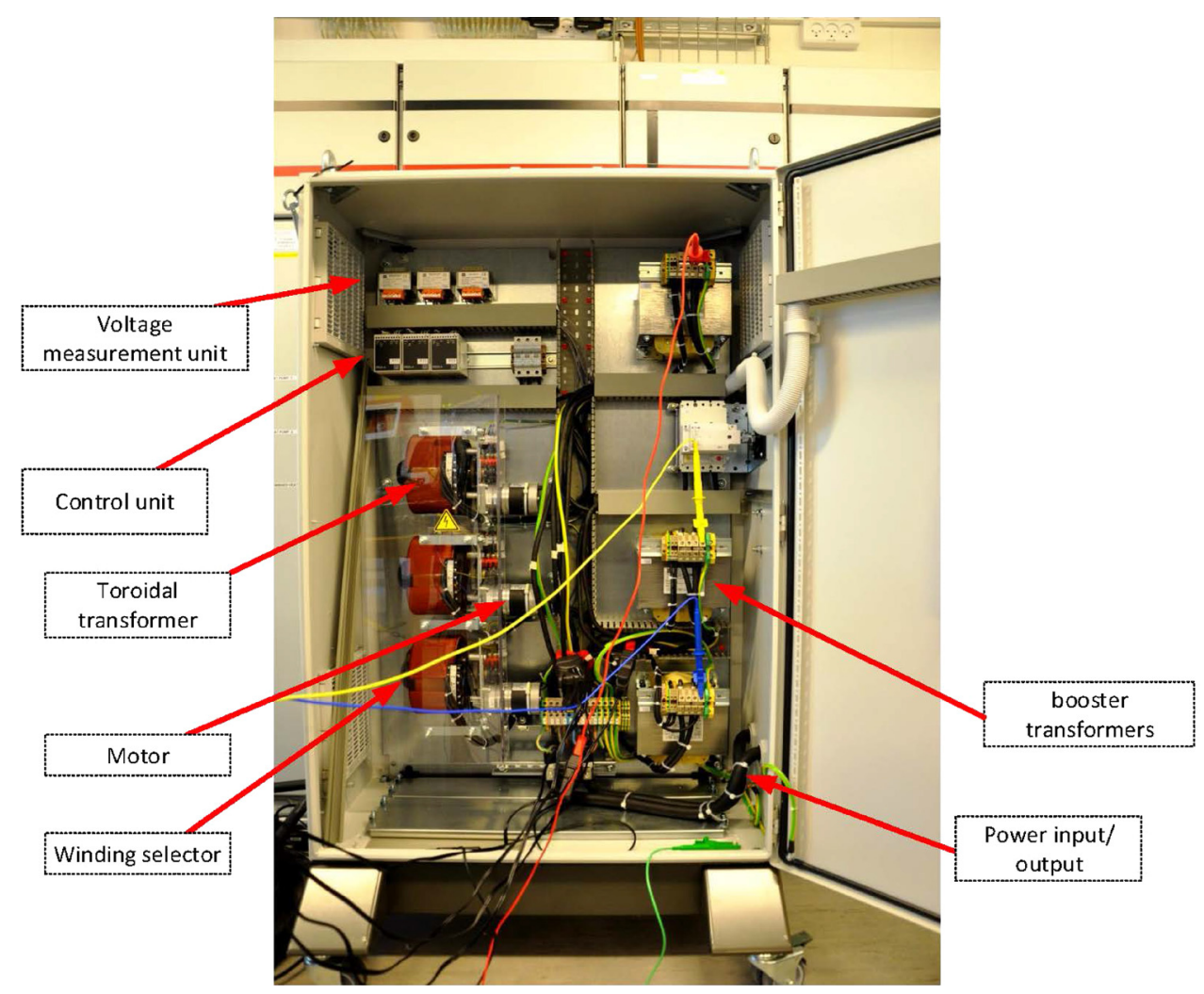

Fig. 15. OLTC device introduction.

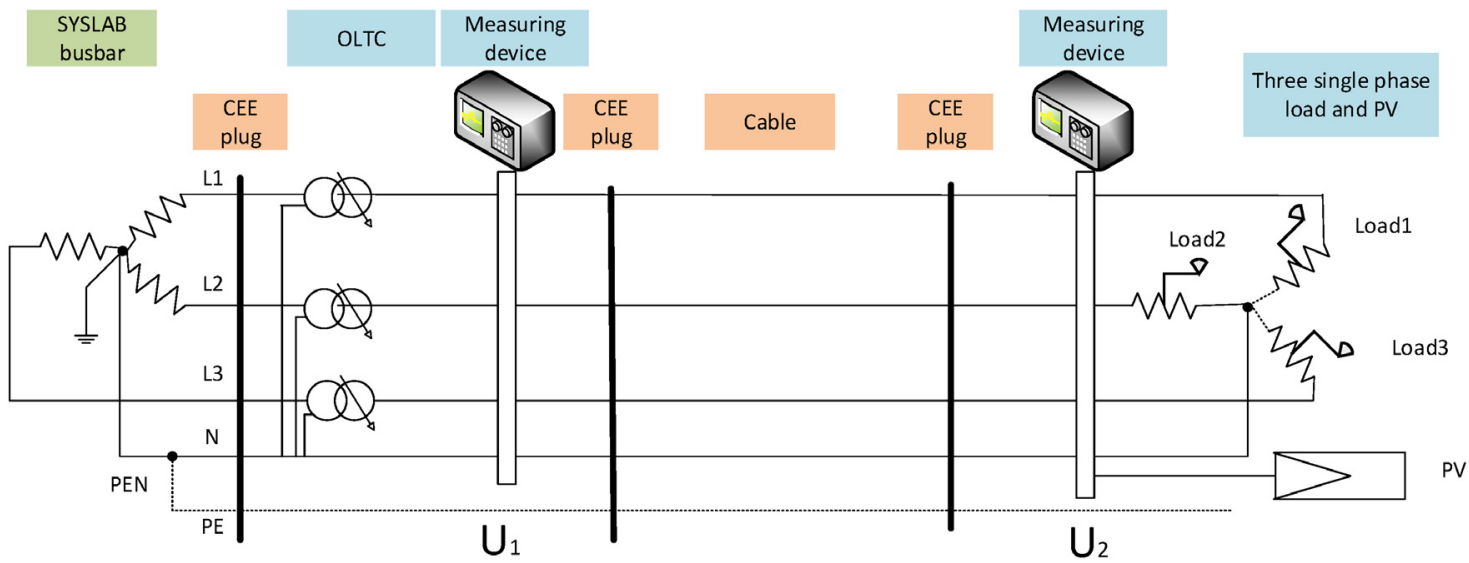

Fig. 16. System layout of testing the operational function of the decoupled three-phase on-load tap changer.

\section{Conclusions}

This paper has presented simulation studies carried out in a Danish EUDP-supported project, where one of the aims is to develop and test the feasibility of a decoupled three-phase on-load tap changer (OLTC) in the distribution system with the objective of improving the distribution network power quality. A real Danish low-voltage network with measurements of the loads and realistic photovoltaic (PV) generation profiles has been used to apply the proposed control. To perform the RMS (root mean square) simulation, the grid elements behavior and the control scheme for the OLTC controller have been modeled with the software DigSilent PowerFactory. In the performed simulation, four indices have been considered when controlling the voltage at the feeder's outermost bus: phase voltage magnitude, voltage unbalance factor, neutral potential, and system losses. The simulation process involved the definition of several PV penetration percentage scenarios, under which five control options for the OLTC have been implemented: no control, three-phase synchronous control, one-phase decoupled control, reactive power provision from photovoltaic inverters, one-phase decoupled control, and reactive power provision of photovoltaic inverters. Under these conditions, the coordinated voltage control of one-phase decoupled OLTC and reactive power provision of PV allowed the improvement of the network's hosting capacity, increasing the acceptable PV penetration from $40 \%$ to $70 \%$. In the process, the voltage unbalance factor was shown to increase with respect to the base case as a result of the opposite trends in the positive and negative sequence magnitudes. In particular, the voltage unbalance growth effect could be even greater if the OLTC control is associated with the reactive power provision from DGs.

The objective of future work is to extend the simulation analysis over a longer time period (e.g., weeks) and to investigate a 
trade-off between the voltage control solutions and the voltage unbalance factors.

\section{Acknowledgments}

The authors would like to thank the financial support of the Danish EUDP program for the project 'Energy Saving by Voltage Management' (under the grant number 5996648995411,). We would also like to thank project partner Ole Michael Pedersen from Dong Energy and Lene Frederiksen, Giovanni Tagliaferri and Kenn Andersen from PSS Energy A/S, Denmark for many interesting discussions.

\section{References}

[1] E. Caamaño-Martín, H. Laukamp, M. Jantsch, T. Erge, J. Thornycroft, H. De Moor, S. Cobben, D. Suna, B. Gaiddon, Interaction between photovoltaic distributed generation and electricity networks, Prog. Photovolt. Res. Appl. 16 (7) (2008) 629-643.

[2] M. Braun, T. Stetz, R. Bründlinger, C. Mayr, K. Ogimoto, H. Hatta, H. Kobayashi, B. Kroposki, B. Mather, M. Coddington, Is the distribution grid ready to accept large-scale photovoltaic deployment? State of the art, progress, and future prospects, Prog. Photovolt. Res. Appl. 20 (6) (2012) 681-697.

[3] T. Stetz, M. Kraiczy, M. Braun, S. Schmidt, Technical and economical assessment of voltage control strategies in distribution grids, Prog. Photovolt. Res. Appl. 21 (6) (2013) 1292-1307.

[4] K. Knezovic, M. Marinelli, R.J. Moller, P.B. Andersen, C. Traholt, F. Sossan, Analysis of voltage support by electric vehicles and photovoltaic in a real Danish low voltage network, in: Power Engineering Conference (UPEC), 49th International Universities, Cluj-Napoca, Romania, IEEE, 2014, pp. 1-6.

[5] T. Hammerschmidt, B. Gwisdorf, T. Borchard, C. Rehtanz, Technical and economic evaluation of voltage regulation strategies for distribution grids with a high amount of fluctuating dispersed generation units, in: 2010 IEEE Conference on Innovative Technologies for an Efficient and Reliable Electricity Supply (CITRES), Waltham, MA, IEEE, 2010, pp. 8-14.

[6] S.B. Kjær, A. Constantin, R.D. Lazar, H.P. Ballegaard, Voltage control in low voltage networks by Photovoltaic inverters-PVNET. Dk, in: EU PVSEC Proceedings, 28th European Photovoltaic Solar Energy Conference and Exhibition, 2012, pp. 3622-3626

[7] R. Caldon, M. Coppo, R. Turri, Distributed voltage control strategy for LV networks with inverter-interfaced generators, Electr. Power Syst. Res. 107 (2014) 85-92.

[8] B. Meersman, B. Renders, L. Degroote, T. Vandoorn, L. Vandevelde, Three-phase inverter-connected DG-units and voltage unbalance, Electr. Power Syst. Res. 81 (4) (2011) 899-906, Apr.

[9] A. Einfalt, F. Kupzog, H. Brunner, A. Lugmaier, Control strategies for smart Low voltage grids - the project DG DemoNet - smart LV grid, in: Integration of Renewables into the Distribution Grid, CIRED 2012 Workshop, Lisbon, IET, 2012, pp. 1-4, 0238.

[10] A. Kechroud, P.F. Ribeiro, W.L. Kling, Distributed generation support for voltage regulation: an adaptive approach, Electr. Power Syst. Res. 107 (2014) 213-220.
[11] N. Jayasekara, P. Wolfs, M.A.S. Masoum, An optimal management strategy for distributed storages in distribution networks with high penetrations of PV, Electr. Power Syst. Res. 116 (2014) 147-157.

[12] Italian reference technical rules for technical standard CEI 0-21, Re connection of active and passive users to the LV electrical utilities, Milano, 2014.

[13] German Technical Standard VDE-AR-N 4105, Power generation systems connected to the low-voltage distribution network, FNNForum Netztechnik/Netzbetrieb im VDE, 2011.

[14] M. Stifter, B. Bletterie, H. Brunner, D. Burnier, H. Sawsan, F. Andren, R. Schwalbe, A. Abart, R. Nenning, F. Herb, DG DemoNet validation: Voltage control from simulation to field test, in: Innovative Smart Grid Technologies (ISGT Europe), 2011 2nd IEEE PES International Conference and Exhibition on, Manchester, IEEE, 2011, pp. 1-8.

[15] F. Kupzog, H. Brunner, W. Pruggler, T. Pfajfar, A. Lugmaier, DG DemoNetConcept-A new algorithm for active distribution grid operation facilitating high DG penetration, Industrial Informatics, 2007 5th IEEE International Conference on, Vienna 2 (2007) 1197-1202.

[16] M. Stifter, R. Schwalbe, W. Tremmel, S. Henein, H. Brunner, B. Bletterie, A. Abart, F. Herb, R. Pointner, DG DemoNet: Experiences from volt/var control field trials and control algorithm advancements, in: 2012 3rd IEEE PES Innovative Smart Grid Technologies Europe (ISGT Europe), IEEE, 2012, pp. 1-7.

[17] M. Stifter, B. Bletterîe, F. Herb, DG DemoNet: Impact of VOLT/VAR control on increasing the voltage band reserve-Results from field trial validations, in: Electricity Distribution (CIRED 2013), 22nd International Conference and Exhibition on, Stockholm, IET, 2013, pp. 1-4, 0508.

[18] I. Leisse, O. Samuelsson, J. Svensson, Coordinated voltage control in medium and low voltage distribution networks with wind power and photovoltaics, in: 2013 IEEE Grenoble Conference, PowerTech, IEEE, 2013, pp. 1-6.

[19] F.A. Viawan, A. Sannino, J. Daalder, Voltage control with on-load tap changers in medium voltage feeders in presence of distributed generation, Electr. Power Syst. Res. 77 (10) (2007) 1314-1322.

[20] F. Bignucolo, R. Caldon, V. Prandoni, Radial MV networks voltage regulation with distribution management system coordinated controller, Electr. Power Syst. Res. 78 (4) (2008) 634-645.

[21] P. Esslinger, R. Witzmann, Regulated distribution transformers in low-voltage networks with a high degree of distributed generation, in: 2012 3rd IEEE PES Innovative Smart Grid Technologies Europe (ISGT Europe), München, Germany, IEEE, 2012, pp. 1-7.

[22] M. Coppo, R. Turri, M. Marinelli, X. Han, Voltage Management in Unbalanced Low Voltage Networks Using a Decoupled Phase-Tap-Changer Transformer, in: Power Engineering Conference (UPEC), 2014 49th International Universities, Cluj-Napoca, Romania, IEEE, 2014, pp. 1-6.

[23] DigSilent, DigSilent technical documentation Two Winding Transformer (3Phase), Gomaringen, Germany, 2010.

[24] DigSilent, DigSilent technical documentation General Load Model, Gomaringen, Germany, 2013.

[25] IEEE Task Force on Load Representation for Dynamic Performance, Load representation for dynamic performance analysis, IEEE Trans. Power Syst. 8 (2) (1993) 472-482.

[26] X. Han, S. You, F.D. Thordarson, V. Tackie, S.M. Ostberg, O.M. Pedersen, H.W Bindner, N.C. Nordentoft, Real-time measurements and their effects on state estimation of distribution power system, in: Innovative Smart Grid Technologies Europe (ISGT EUROPE), 2013 4th IEEE/PES, Copenhagen, IEEE, 2013, pp. $1-5$.

[27] P. Pillay, M. Manyage, Definitions of voltage unbalance, IEEE Power Eng. Rev (2001) 50-51. 